\title{
On the Verification of Memory Management Mechanisms
}

\author{
Iakov Dalinger* Mark Hillebrand* Wolfgang Paul \\ January 2005 \\ Id: verificationmm.tex,v 1.42 2005/02/18 17:10:17 mah Exp
}

\begin{abstract}
We define physical machines as processors with physical memory and swap memory; in user mode physical machines support address translation. We report about the formal verification of a complex processor supporting address translation by means of a memory management unit (MMU). We give a paper and pencil proof that physical machines together with appropriate page fault handlers simulate virtual machines.
\end{abstract}

\section{Contents}

1 Introduction $\quad 2$

1.1 The challenge of verifying entire systems . . . . . . . . 2

1.2 Overview of this paper . . . . . . . . . . . . 3

1.3 Related work . . . . . . . . . . . . . . . . 3

2 Virtual machines $\quad 3$

2.1 Notation . . . . . . . . . . . . . . . 3

2.2 Specifying the instruction set architecture . . . . . . 4

2.3 Interrupts . . . . . . . . . . . . . . . . 6

3 Physical machines $\quad 7$

3.1 Address translation . . . . . . . . . . . . . . . 8

3.2 Modeling an $\mathrm{I} / \mathrm{O}$ device . . . . . . . . . . . . . . 9

4 Construction and local correctness of MMUs 9

4.1 Notation . . . . . . . . . . . . . . . . . . . 9

4.2 Memory interface . . . . . . . . . . . . . . 9

4.3 MMU construction and operating conditions . . . . . . . . 11

4.4 Local MMU correctness . . . . . . . . . . . . . . . . . . 12

5 Guaranteeing the operating conditions 13

5.1 Software Synchronization Convention . . . . . . . . . . . 14

5.2 Hardware mechanisms for synchronization . . . . . . . . . 14

\footnotetext{
${ }^{*}$ Work partially funded by the German Federal Ministry of Education, Science, Research and Technology (BMBF) in the framework of the Verisoft project under grant 01 IS C38. Work of the second author was also partially funded by IBM Entwicklung GmbH Boeblingen. The responsibility for this article lies with the authors.
} 
6 Processor correctness $\quad 15$

6.1 Correctness criteria . . . . . . . . . . . . 15

6.2 Correctness proof with external interrupt signals . . . . . 17

6.3 Correctness proof . . . . . . . . . . . . 17

7 Virtual machine simulation $\quad 19$

7.1 Memory map of the physical machine . . . . . . . . . 19

7.2 Simulation relation . . . . . . . . . . . . . . 20

7.3 Page fault handler and software conditions . . . . . . . . . 21

7.4 Simulation theorem . . . . . . . . . . . . . 22

8 Summary and further work

\section{Introduction}

\subsection{The challenge of verifying entire systems}

In the spirit of the famous CLI stack [Boy89] the research of this paper aims at the formal verification of entire computer systems consisting of hardware, compiler, operating system, communication system, and applications. Working with the Boyer-Moore theorem prover [BM88] the researchers of the CLI stack project succeeded as early as 1989 to prove formally the correctness of a system which contained among others the following components: (i) a non pipelined processor [Hun89], (ii) an assembler [Moo89], (iii) a compiler for an imperative language [You89] with the only data type int, assignments, if then else, while loops, and function calls, (iv) a rudimentary operating system kernel [Bev89] written in machine language. This kernel provides scheduling for a fixed number of processes; each process has the right to access a fixed interval of addresses in the processors physical memory. An attempt to access memory outside these bounds leads to an interrupt. Interprocess communication and system calls apparently were not provided.

In the years from 1989 to 2002 to the best of our knowledge no verification project aiming at the formal verification of entire computer systems was started anywhere. In [Moo03] the principal investigator of the CLI stack project J S. Moore declares the design and formal verification of practical embedded systems 'from the transistor level to the software' a grand challenge problem. A central goal of the Verisoft project [Ver03], funded by the federal German Government and started in 2003, is to solve this grand challenge problem.

This paper makes two necessary steps towards the verification of entire complex systems. (i) We report about the formal verification of a processor with memory management units (MMUs). MMUs provide hardware support for address translation; address translation is needed for the implementation of address spaces provided by modern operating systems. (ii) We present a paper and pencil correctness proof for a virtual memory emulation based on a very simple page fault handler. As the formal treatment of I/O-devices is an open problem we have to state the correctness of a driver for the swap memory as an axiom.

In subsequent papers we will address the verification of a compiler for a C-like language with in-line assembler code and of an operating system kernel. For preliminary versions of these results see [?, ?, ?]. 


\subsection{Overview of this paper}

In Section 2 we briefly review the standard formal definition of the DLX instruction set architecture (ISA) for virtual machines. We put emphasis on the handling of internal and external interrupts. In Section 3 on physical machines we enrich the ISA by the standard mechanisms for operating system support: (i) user mode / system mode and (ii) address translation in user mode. In Section 4 we present a (non-optimized) construction of an MMU and prove its correctness under nontrivial operating conditions. Note that in pipelined processors separate MMUs are used for instruction fetch and load / store. In Section 5 we show how the operating conditions for both MMUs can be guaranteed by a combination of hardware mechanisms and software conventions. Section 6 gives the main new arguments of the processor correctness proof assuming that the software conventions are met. In Section 7 we present a simple page fault handler which obeys the software convention. We show that a physical machine with this page fault handler emulates a virtual machine. In Section 8 we present conclusions and further work.

\subsection{Related work}

The processor verification presented here extends work on the VAMP processor presented in $\left[\mathrm{BJK}^{+} 03\right.$, Bey04]. The treatment of external interrupts is in the spirit of [SH98, MP00]. Formal proofs are in PVS [OSR92] and - except for some limited use of PVS's model checker - interactive. We stress that some central lemmas in $\left[\mathrm{SH} 98, \mathrm{BJK}^{+}\right.$03] (e.g. correctness of Tomasulo schedulers) have similar counterparts, which can be proven using the very rich set of automatic methods for hardware verification (e.g. [?]). How to profit from these automatic methods in correctness proofs of entire processors continues to be an amazingly difficult topic of research. Some recent progress is reported in [ACHK04].

As for the new results of this paper: we are not aware of previous work on the verification of MMUs. We are also not aware of previous theoretical work on the correctness of virtual memory emulations.

\section{Virtual machines}

\subsection{Notation}

We denote the concatenation of bit strings $a \in\{0,1\}^{n}$ and $b \in\{0,1\}^{m}$ by $a \circ b$. For bits $x \in\{0,1\}$ and nonnegative natural numbers $n \in \mathbb{N}^{+}$ we define inductively $x^{1}=x$ and $x^{n}=x^{n-1} \circ x$. Thus, for instance $0^{5}=00000$ and $1^{2}=11$.

Overloading symbols like,$+ \cdot$, and $<$ we will allow arithmetic on bit strings $a \in\{0,1\}^{n}$. In these cases arithmetic is binary modulo $2^{n}$. We will consider $n=32$ for addresses / register contents and $n=20$ for page indices.

We model memories $m$ as mappings from addresses $a$ to values $m(a)$. For natural numbers $d$ we denote by $m_{d}(a)$ the content of $d$ consecutive memory cells starting at address $a$ :

$$
m_{d}(a)=m(a+d-1) \circ \cdots \circ m(a)
$$

Page size will be $4 K=2^{12}$. We split virtual addresses $v a=v a[31: 0]$ into page index va.px $=v a[31: 12]$ and byte index $v a . b x=v a[11: 0]$. 


\begin{tabular}{|l|l|l|}
\hline Address & Synonym & Meaning \\
\hline \hline 00000 & SR & Status register \\
\hline 00001 & ESR & Exception status register \\
\hline 00010 & ECA & Exception cause register \\
\hline 00011 & EPC & Exception PC \\
\hline 00100 & EDPC & Exception DPC \\
\hline 00101 & Edata & Exception data \\
\hline 00110 & RM & Rounding mode \\
\hline 00111 & IEEEf & IEEE flags \\
\hline 01000 & FCC & Floating point condition code \\
\hline 01001 & pto & Page table origin \\
\hline 01010 & ptl & Page table length \\
\hline 01011 & EMODE & Exception mode \\
\hline 10000 & MODE & Mode \\
\hline
\end{tabular}

Table 1: Special purpose registers. Indices 01100 to 01111 are not assigned.

Thus, $v a=v a . p x \circ v a . b x$.

\subsection{Specifying the instruction set architecture}

Virtual machines are the hardware model visible for user processes. Important parameters of such a machine are the following:

- The number $V$ of pages of accessible virtual memory. This defines the set of accessible virtual addresses

$$
V A=\{a \mid 0 \leq a<V \cdot 4 K\}
$$

- The number $e \in \mathbb{N}$ of external interrupt signals.

- The status register $S R \in\{0,1\}^{32}$. This is the vector of mask bits for the interrupts. In physical machines it comes from the status register.

- The set $V S A \subseteq\{0,1\}^{5}$ of addresses of user visible special purpose registers. Table 1 shows the entire set of special purpose registers, that will be visible for a physical machine. For the virtual machine only the registers $R M, I E E E f$, and $F C C$ will be visible. Hence $V S A=\{00110,00111,01000\}$.

Formally, the configurations of a virtual machine is a 6-tuple

$$
c_{V}=\left(c_{V} \cdot P C, c_{V} \cdot D P C, c_{V} \cdot G P R, c_{V} \cdot S P R, c_{V} \cdot v m, c_{V} \cdot p\right) \text {. }
$$

The individual components are:

- the program counter $c_{V} \cdot P C \in\{0,1\}^{32}$ and the delayed program counter $c_{V} . D P C \in\{0,1\}^{32}$. Both are used to implement the delayed branch mechanism (see Chapter 4 in [MP00] for details);

- The general purpose register file $c_{V} \cdot G P R:\{0,1\}^{5} \rightarrow\{0,1\}^{32}$. Registers are 32 bits long, register 0 always contains $0^{32}$.

- The special purpose register file $c_{V} \cdot S P R: V S A \rightarrow\{0,1\}^{32}$. We also refer to special purpose register by $c_{V} \cdot x=c_{V} \cdot S P R[x]$ where $x$ is one of the synonyms from Table 1. 
- The byte addressable virtual memory $c_{V} . v m: V A \rightarrow\{0,1\}^{8}$.

- The write protection function $c_{V} \cdot p:\{v a . p x \mid v a \in V A\} \rightarrow\{0,1\}$. Virtual addresses on the same page have the same protection bit.

Let $C_{V}$ be the set of virtual machine configurations. An instruction set architecture (ISA) is formally specified as a transition function $\delta_{V}: C_{V} \times$ $\{0,1\}^{e} \rightarrow C_{V}$ mapping configurations $c_{V} \in C_{V}$ and a vector of external event signals eev $\in\{0,1\}^{e}$ to a next configuration $c_{V}^{\prime}=\delta_{V}\left(c_{V}, e e v\right)$. For the DLX instruction set we outline the formal definition of this function with an emphasis on the treatment of interrupts.

The instruction to be executed in configuration $c_{V}$ is found in the four bytes in virtual memory starting at the address of the delayed PC

$$
I\left(c_{V}\right)=c_{V} \cdot v m_{4}\left(c_{V} \cdot D P C\right)
$$

The opcode consists of the leading six bits of the instruction

$$
o p c\left(c_{V}\right)=I\left(c_{V}\right)[31: 26]
$$

The type of an instruction determines, how the bits outside the opcode are interpreted. If the opcode consists of all zeros we have for instance an R-type instruction

$$
R \text {-type }\left(c_{V}\right)=\left(\operatorname{opc}\left(c_{V}\right)=000000\right)
$$

Other instruction types are defined in a similar way. Many instructions can be decoded just from the opcode, e.g. a load word instruction is recognized by

$$
l w\left(c_{V}\right)=\left(\operatorname{opc}\left(c_{V}\right)=100011\right)
$$

For R-type instructions one has to refer to a secondary opcode. Depending on the instruction type the register destination address is found at different positions in the instruction

$$
R D\left(c_{V}\right)= \begin{cases}I\left(c_{V}\right)[15: 11] & \text { if } R \text {-type }\left(c_{V}\right) \\ I\left(c_{V}\right)[20: 16] & \text { otherwise }\end{cases}
$$

In a similar way one can define register source addresses $R S 1\left(c_{V}\right), R S 2\left(c_{V}\right)$, the sign extended immediate constant $\operatorname{simm}\left(c_{V}\right)$, etc. The effective address of a load or store instruction is computed as the sum of the general purpose register addressed by $R S 1\left(c_{V}\right)$ and the sign extended immediate constant

$$
e a\left(c_{V}\right)=c_{V} \cdot G P R\left(R S 1\left(c_{V}\right)\right)+\operatorname{simm}\left(c_{V}\right)
$$

A load word instruction stores four bytes of virtual memory starting at address $e a\left(c_{V}\right)$ into the general purpose register addressed by $R S 1\left(c_{V}\right)$. This can be expressed by equations like

$$
l w\left(c_{V}\right) \rightarrow\left(c_{V}^{\prime} \cdot \operatorname{GPR}\left(R S 1\left(c_{V}\right)\right)=c_{V} \cdot v m_{4}\left(e a\left(c_{V}\right)\right)\right) .
$$

Components of the configuration that are not mentioned on the righthand side of the implication are meant to be unchanged. This definition however ignores both internal and external interrupts; therefore even for virtual machines it is an oversimplification. 


\begin{tabular}{|l|l|l|l|l|}
\hline Index $j$ & Symbol & Meaning & Maskable & External \\
\hline \hline 0 & reset & Reset & No & Yes \\
\hline 1 & ill & Illegal instruction & No & No \\
\hline 2 & mal & Misaligned access & No & No \\
\hline 3 & pff & Page fault during fetch & No & No \\
\hline 4 & pfls & Page fault during load / store & No & No \\
\hline 5 & trap & Trap & No & No \\
\hline 6 & xovf & Fixed point overflow & Yes & No \\
\hline 7 & fovf & floating point (FP) overflow & Yes & No \\
\hline 8 & funf & FP underflow & Yes & No \\
\hline 9 & finx & FP inexact result & Yes & No \\
\hline 10 & fdbz & FP division by zero & Yes & No \\
\hline 11 & finv & FP invalid operation & Yes & No \\
\hline 12 & ufop & Unimplemented FP operation & No & No \\
\hline 13 & timer & Timer & No & No \\
\hline$j>13$ & $i o[j]$ & Interrupt from I/O device $j-13$ & Yes & Yes \\
\hline
\end{tabular}

Table 2: Interrupts

\section{$2.3 \quad$ Interrupts}

We define below a predicate $\operatorname{JISR}\left(c_{V}\right.$, eev) (jump to interrupt service routine) depending on both the current configuration $c_{V}$ and the current values eev $\in\{0,1\}^{e}$ of the external interrupt event signals. Only if this signal stays inactive does the above equation hold

$$
\left(\neg J I S R\left(c_{V}, e e v\right) \wedge l w\left(c_{V}\right)\right) \rightarrow\left(c_{V}^{\prime} \cdot G P R\left(R S 1\left(c_{V}\right)\right)=c_{V} \cdot v m_{4}\left(e a\left(c_{V}\right)\right)\right)
$$

An activation of the JISR signal has for physical machines a well defined effect on the program counters and on the special purpose registers. The effect on virtual machine computations however is that control is handed over to the operating system kernel. This effect can only be defined in a model, which includes the operating system kernel too. ${ }^{1}$ For the same reason rfe-instructions (return from interrupt) are illegal for virtual machines.

For the definition of signal $\operatorname{JISR}\left(c_{V}, e e v\right)$ for physical machines, we consider the 32 interrupts from Table 2 with indices $j \in I P=\{0, \ldots, 31\}$. For virtual machines we ignore page fault interrupts, thus we only consider $j \in I V=I P \backslash\{3,4\}$.

The activation of signal $J I S R\left(c_{V}, e e v\right)$ can be caused by the activation of external interrupt lines eev[j] or by internal interrupt event signal $i e v\left(c_{V}\right)[j]$. We define the cause vector by

$$
c a\left(c_{V}, e e v\right)[j]= \begin{cases}\operatorname{eev}[0] & \text { if } j=0 ; \\ \operatorname{eev}[j-13] & \text { if } j \text { external, } j \neq 0 \\ \operatorname{iev}\left(c_{V}\right)[j] & \text { otherwise. }\end{cases}
$$

Formally, external interrupts lines are external input signals for the next state computation. Internal interrupt event signals can be defined as functions of the current configuration. A straightforward definition of the

\footnotetext{
${ }^{1}$ See the lecture notes [SysArch04] (in German) for details.
} 
misalignment signal would for instance include terms like

$$
\begin{aligned}
\operatorname{mal}\left(c_{V}\right)=\operatorname{iev}\left(c_{V}\right)[2]= & \left(c_{V} \cdot D P C[1: 0] \neq 00\right) \vee \\
& \left(l w\left(c_{V}\right) \wedge\left(e a\left(c_{V}\right)[1: 0] \neq 00\right)\right) \vee \cdots
\end{aligned}
$$

For virtual machines, but not for physical machines, an attempt to read or write special purpose registers other than $R M$, IEEEf, FCC is illegal. Reading or writing these registers is achieved with commands movi2s or movs $2 i$; special purpose registers are addressed with instruction field $S A\left(c_{V}\right)=I\left(c_{V}\right)[10: 6]$. Thus a straightforward formal definition of the illegal instruction signal would include a term like

$$
\begin{aligned}
i l l\left(c_{V}\right) & =i e v\left(c_{V}\right)[1] \\
& =\left(\left(\operatorname{movi} 2 s\left(c_{V}\right) \vee\left(\operatorname{movs} 2 i\left(c_{V}\right)\right) \wedge\left(S A\left(c_{V}\right) \notin V S A\right) \vee \cdots\right.\right.
\end{aligned}
$$

The cause vector $c a\left(c_{V}, e e v\right)$ is ANDed bit wise with a mask vector

$$
\operatorname{mask}\left(c_{V}\right)[j]= \begin{cases}S R[j] & \text { if } j \text { maskable } \\ 1 & \text { otherwise }\end{cases}
$$

in order to obtain the masked cause vector:

$$
m c a\left(c_{V}, e e v\right)[j]=c a\left(c_{V}, e e v\right) \wedge \operatorname{mask}\left(c_{V}\right)[j]
$$

The interrupt occurs if at least one masked cause bit is on

$$
J I S R\left(c_{V}, e e v\right)=\bigvee_{j \in I V} m c a\left(c_{V}, e e v\right)[j]
$$

Thus, although a virtual machine cannot read or write the status register $S R$, this register is nevertheless visible via the masked cause vector and the $J I S R$-signal.

\section{Physical machines}

Physical machines are the sequential programming model of the hardware as seen by the programmer of an operating system kernel. Compared with the ISA of the virtual machine, more details are visible in configurations $c_{P} \in C_{P}$ of physical machines.

- All special purpose registers from Table 1 are visible. Formally $c_{P} . S P R: P S A \rightarrow\{0,1\}^{32}$ with $P S A=\left\{\operatorname{bin}_{5}(a): a \leq 13\right\}$ where $\operatorname{bin}_{n}(a) \in\{0,1\}^{n}$ is the bitvector of length $n$ such that $\left\langle\operatorname{bin}_{n}(a)\right\rangle=a$. The mode register $c_{P} . S P R[10000]=c_{P}$. mode distinguishes between system mode $\left(c_{P}\right.$.mode $\left.[0]=0\right)$ and user mode. In system mode reading and writing any special purpose register is legal.

- Page faults are visible, thus in the definition of the JISR-signal the full set of indices $I P$ is used instead of $I V$.

- For physical machines the next state $\delta_{P}\left(c_{P}, e e v\right)$ is defined in the case of an active signal $J I S R\left(c_{P}, e e v\right)$. See [MP00] for details. Similarly, in system mode physical machines can legally execute an rfe (return from exception) instruction.

- Instead of a uniform virtual memory the user now sees two memories: physical memory $c_{P} . p m$ and swap memory $c_{P} . s m$. The number of pages of available physical memory is specified by a parameter $P$. 


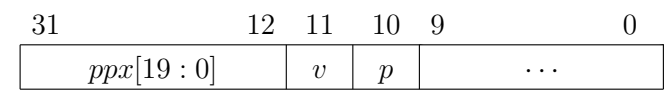

Figure 1: Page Table Entry

- In user mode accesses to physical memory are translated.

In the remainder of this section we first specify a 1-level translation mechanism and the corresponding internal interrupt event signals $p f f\left(c_{P}\right)$ and $p f l s\left(c_{P}\right)$. Then we model I/O operations with the swap memory.

\subsection{Address translation}

In user mode, i.e. if $c_{P}$.mode $=1$, virtual addresses $v a=v a . p x \circ v a . b x$ are transformed into three signals $p m a\left(c_{P}, v a\right), p f f\left(c_{P}\right), p f l s\left(c_{P}\right)$, where $p f f$ and $p f l s$ are interrupt event signals and $p m a$ is the physical memory address in case the page fault signals stay inactive. Memory region $c_{P} . p m_{c_{P} . p t l \cdot 4+4}\left(c_{P} . p t o \cdot 4 K\right)$ is interpreted as the current page table. The page table entry address for virtual address $v a$ is defined as

$$
\text { ptea }\left(c_{P}, v a\right)=c_{P} \cdot p t o \cdot 4 K+4 \cdot v a \cdot p x
$$

and the corresponding page table entry is

$$
p t e\left(c_{P}, v a\right)=c_{P} \cdot p m_{4}\left(p t e a\left(c_{P}, v a\right)\right) .
$$

These functions are only defined if there is no page table length exception

$$
p t l e x c p\left(c_{P}, v a\right)=\left(\langle v a \cdot p x\rangle>\left\langle c_{P} \cdot p t l\right\rangle\right)
$$

From the page table entry we extract the physical page index $p p x$, the valid bit $v$, and the protection bit $p$ as suggested in Figure 1 by

$$
\begin{aligned}
p p x\left(c_{P}, v a\right) & =p t e\left(c_{P}, v a\right)[31: 12] \\
v\left(c_{P}, v a\right) & =p t e\left(c_{P}, v a\right)[11] \\
p\left(c_{P}, v a\right) & =p t e\left(c_{P}, v a\right)[10]
\end{aligned}
$$

In case the valid bit is on, the physical memory address $p m a$ is obtained by concatenation of the physical page index with the byte index $v a . b x$ :

$$
p m a\left(c_{P}, v a\right)=p p x\left(c_{P}, v a\right) \circ v a . b x
$$

A page fault on fetch occurs, if page table length exception or invalid access occurs with virtual address $d p c$

$$
p f f\left(c_{P}\right)=\operatorname{ptlexcp}\left(c_{P}, c_{P} . D P C\right) \vee \neg v\left(c_{P}, c_{P} . D P C\right)
$$

In the absence of page faults on fetch we have in user mode now

$$
I\left(c_{P}\right)=c_{P} \cdot m_{4}\left(p m a\left(c_{P}, c_{P} \cdot D P C\right)\right)
$$

In the absence of page faults on fetch a page fault on load store concerns virtual address $e a\left(c_{P}\right)$. Besides page table length exceptions and invalid access there might also be an attempt to perform a store operation, indicated by predicate $s\left(c_{P}\right)$, to a write protected page

$$
p f l s\left(c_{P}\right)=p t l e x c p\left(c_{P}, e a\left(c_{P}\right)\right) \vee \neg v\left(c_{P}, e a\left(c_{P}\right)\right) \vee s\left(c_{P}\right) \wedge p\left(e a\left(c_{P}\right)\right)
$$

It is not difficult to specify multi-level translation can be formally specified in a similar way, see e.g. [Hil05, Chapter 5]. 


\subsection{Modeling an I/O device}

In order to handle page faults, one clearly has to be able to exchange pages between the physical memory $c_{P} . p m$ and the swap memory $c_{P} . s m$. For a (minimal) detailed treatment of this process one has to do four things:

1. Define I/O-ports as a portion of memory shared between the CPU and the I/O device holding the swap memory.

2. Specify the detailed protocol of the I/O-devices.

3. Construct a driver program say with the following three parameters passed on fixed addresses $a_{1}, a_{2}, a_{3}$ in physical memory: a physical page index parameter $\operatorname{ppxp}\left(c_{P}\right)=c_{P} \cdot p m_{4}\left(a_{1}\right)$, a swap memory page index parameter $\operatorname{spxp}\left(c_{P}\right)=c_{P} \cdot p m_{4}\left(a_{2}\right)$, and a physical-toswap flag $p 2 s\left(c_{P}\right)=c_{P} \cdot p m\left(a_{3}\right)$ indicating, whether a page is to be transferred from physical to swap memory $(p 2 s=1)$ or vice versa.

4. Show (among other things): ${ }^{2}$ if the driver is started in configuration $c_{P}$ and never interrupted, then it eventually terminates in configuration $c_{P}^{\prime}$ with

$$
\begin{aligned}
& c_{P}^{\prime} \cdot s m_{4 K}\left(\operatorname{spxp}\left(c_{P}\right) \cdot 4 K\right)=c_{P} \cdot p m_{4 K}\left(p p x p\left(c_{P}\right) \cdot 4 K\right) \text { if } p 2 s\left(c_{P}\right)=1 \\
& c_{P}^{\prime} \cdot p m_{4 K}\left(p p x p\left(c_{P}\right) \cdot 4 K\right)=c_{P} \cdot s m_{4 K}\left(\operatorname{spxp}\left(c_{P}\right) \cdot 4 K\right) \text { if } p 2 s\left(c_{P}\right)=0
\end{aligned}
$$

Without this detailed treatment of I/O devices we have to assume the existence of a correct driver program as an axiom.

\section{Construction and local correctness of MMUs}

\subsection{Notation}

For cycles $t$ and hardware signals or register contents $x$ we denote by $x^{t}$ the value of $x$ during cycle $t$. We will refer to the hardware configuration by $h$. The components of this configuration are registers $h . R$ or memories h.mem. We often abbreviate $h . x$ by $x$.

\subsection{Memory interface}

We construct MMUs for processors with two first level caches, an instruction cache $C I$ for fetches and a data cache $C D$ for load / store instructions. Therefore the CPU communicates with the memory system via two sets of busses: one connecting the CPU with the instruction cache and the other one with the data cache (see Figure 2). We assume that the same protocol is used on both busses. Examples of the protocol are shown in Figure 3 for an instruction fetch with and without a cache hit. The essential properties of the bus protocol and the memory system are the following:

1. Accesses last from the activation of a read or write request signal (in the example $m r$ ) until one cycle after the busy signal is turned off; if the busy signal is not turned on at all, accesses last a single cycle.

\footnotetext{
${ }^{2}$ These are: (i) prove that program control returns (e.g. in case a jump and link instruction was used to call the driver $\left.c_{P}^{\prime} \cdot D P C=c_{P} \cdot G P R[11111], c_{P}^{\prime} \cdot P C=c_{P} \cdot G P R[11111]+4\right)$, (ii) prove that except for appropriately defined book keeping information no other parts of the configuration changed, and (iii) the driver never leaves its own code region (needed to prove the correctness of the driver arguing only about the code of the driver).
} 


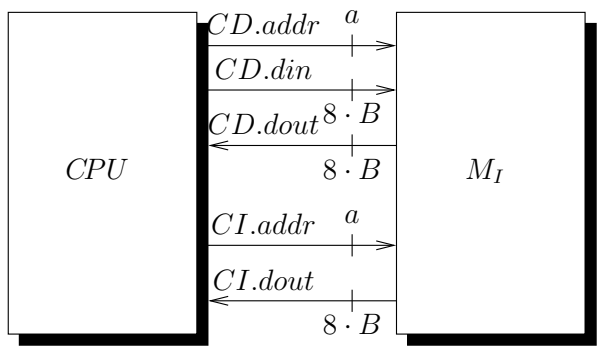

Figure 2: Old Memory Interface without MMUs
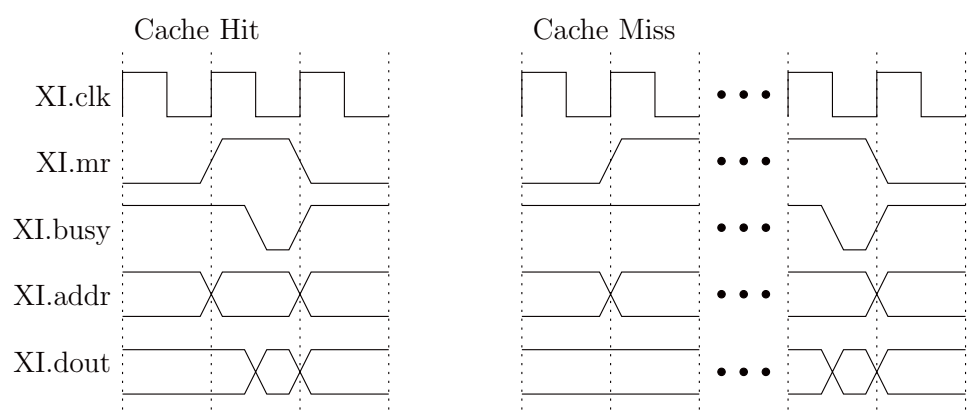

Figure 3: Timing Diagrams for Fetch Operations

2. Read and write requests are not allowed to be given simultaneously.

3. For the duration of an access, inputs from the CPU to the memory system must be held constant.

4. Liveness: if Conditions 2 and 3 are fulfilled, every access eventually ends.

5. Shared memory semantics: for cycles $t$ and addresses $a$ define last $(a, t)$ as the last cycle $t^{\prime}$ before $t$, when a write access to address $a$ (necessarily on the data cache via bus $C D$.din) ended. Now assume a read access to cache $X \in\{C I, C D\}$ with address $a$ ends in cycle $t$. Then the result on bus $X$.dout is

$$
X . d o u t^{t}=C D \cdot \operatorname{din}^{\text {last }(a, t)}
$$

The definition permits to define the state of the two port memory system memory system $m(h)$ at time $t$ by

$$
m(h)^{t}(a)=C D \cdot \operatorname{din}^{t} l a s t(a, t)
$$

For a formal and complete version of this definition ${ }^{3}$ see pages .... of [Bey04]. For a construction of a split cache system and a transcript of a formal proof, that it satisfies this specification, see [Bey04], pages 1-110. Guaranteeing that the CPU keeps inputs constant (Condition 3) during all accesses requires the construction of so-called stabilizer circuits, both for the instruction port and for the data port of the memory system. These circuits are needed for instance, if a fetch is in progress and simultaneously

${ }^{3}$ last does not always exist 


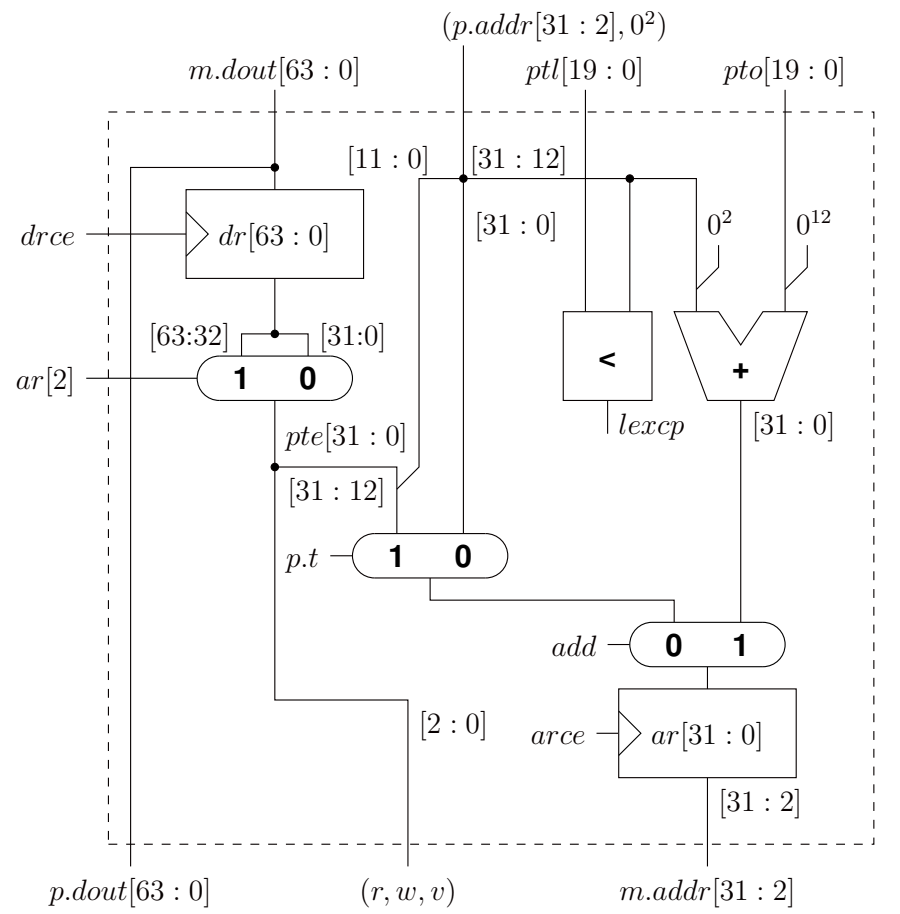

Figure 4: MMU Datapaths.

special addresses are forced into the $P C$ and $D P C$ due to an interrupt recognized deeper down in the pipeline. In this case the started access is completed with latched versions of the $P C$ and $D P C$. If this precaution is not taken, liveness (Condition 4) is not guaranteed. For details see Chapter 4.4 of [Bey04].

\subsection{MMU construction and operating conditions}

Figures 4 and 5 show datapaths and control automaton of a straightforward non optimized construction of an MMU. Two copies of this MMU are placed between the CPU and the two caches as shown in Figure 6 . Note that the interface to the memory system is eight bytes wide and the address width is only 29 bits.

In system mode this MMU will only perform address translation under non trivial operating conditions. Consider an access of the CPU to the MMU lasting from a start cycle $t s$ to an end cycle $t e>t s$. We have to require that no signal or register content $x$ from the four groups listed below changes its value during the access, so for all $\in\{t s, \ldots, t e\}$ we have $x^{t}=x^{t s}$.

G1. Inputs from the CPU to the interface busses of the MMU; these are p.dout, p.addr, p.mr, and p.mw.

G2. The CPU registers h.mode, h.pto, and h.ptl relevant for translation.

G3. In case of a translated accessing the page table entry used for translation, the shared memory content $m(h)_{4}($ ptea $)$ with ptea $=$ h.pto . $4096+4 \cdot p . a d d r^{t s} \cdot p x$. 


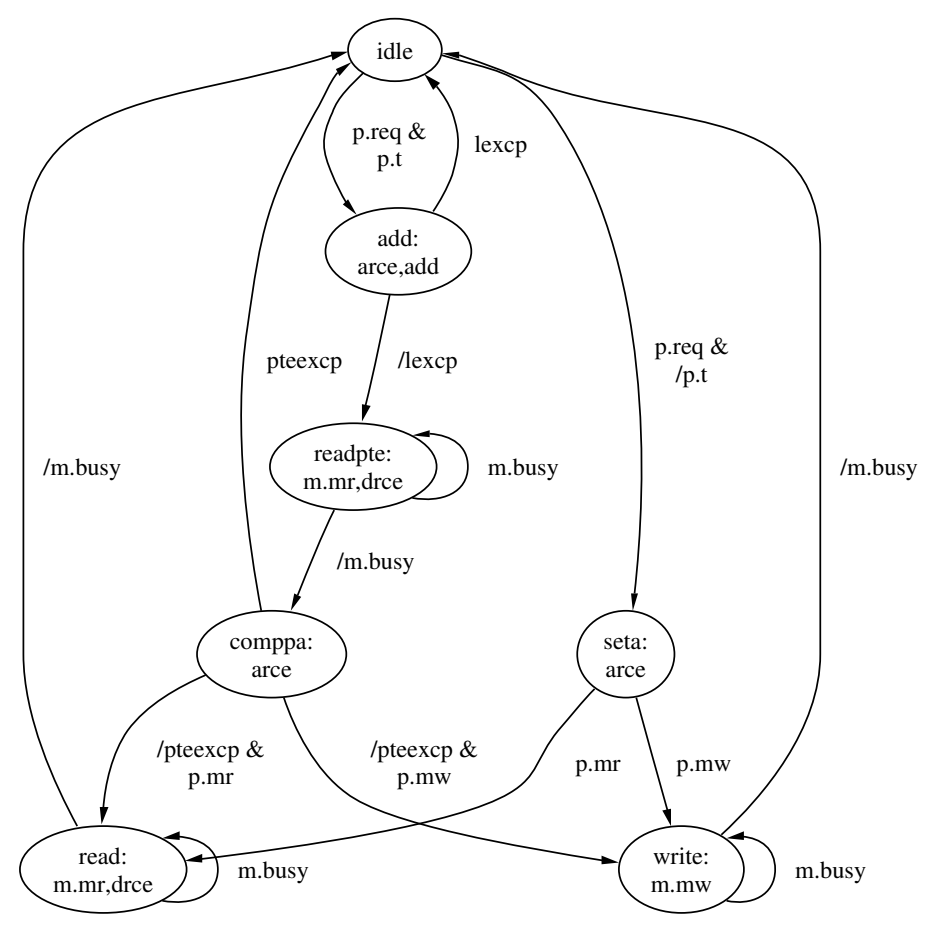

Figure 5: MMU Control Automaton. The signal p.req $=p . m w \vee p . m r$ indicates an ongoing request.

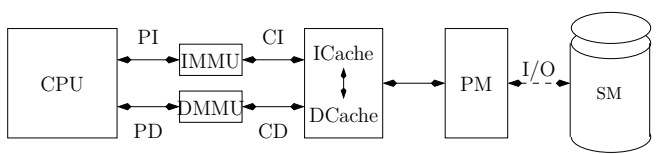

Figure 6: Processor and MMUs

G4. In case of read access with physical address $p a$, the shared memory content $m(h)_{8}(p a)$.

Using definitions analogous to Section 3.1 one can define for hardware configurations $h$ and virtual addresses $v a$ a page table entry address ptea $(h, v a)$ a page table entry pte $(h, v a)$ and a physical memory address pma $(h, v a)$. Note that under the operating conditions the virtual address $v a$, the translation $p m a(h, v a)$ and in case of a read the data read from the shared memory stay the same during the whole access.

Assuming these operating conditions, we outline a fairly straightforward correctness proof for the MMU in the next subsection. Guaranteeing the operating conditions will be a considerably tougher issue.

\subsection{Local MMU correctness}

There is an obvious case split on the kind of access (i) read / write (ii) translated / untranslated (iii) with / without exception. We treat 
here only the case of a translated read access without exception.

First we identify the sequence of states in the control automaton for such a request. For states $s$ we denote by $s^{+}$the fact that control stays in state $s$ until the busy signal is taken away from the memory interface.

Lemma 1 (Path lemma) In a translated read without exception the path through the control automaton is

$$
\text { idle } \rightarrow \text { add } \rightarrow \text { readpte }^{+} \rightarrow \text { comppa } \rightarrow \text { read }^{+} \rightarrow \text { idle } .
$$

Lemma 2 (Local correctness) The result p.din ${ }^{\text {te }}$ of a translated read without exception to a virtual address supplied as va $=$ p.addr ${ }^{t s}$ is

$$
\left.p \cdot d i n^{t e}=m\left(h^{t s}\right)_{8}\left(p m a\left(h^{t s}, v a \circ 0^{3}\right)\right)\right) .
$$

Proof. In cycle $t s+1$ control is in state $a d d$. Hence in cycle $t s+2$ we have in the MMU's address register ar the address of the required page table entry

$$
\begin{aligned}
a r^{t s+2} & =h \cdot p t o^{t s+1}+4 \cdot p \cdot a d d r^{t s+1} \cdot p x \\
& =h \cdot p t o^{t s}+4 \cdot p \cdot a d d r^{t s} \cdot p x
\end{aligned}
$$

by $G 1$ and $G 2$. Consider the cycle $t^{\prime} \geq t s+3$ when control is in state readpte and the busy signal from the memory interface is zero. Using $G 3$ one argues that the MMU's data register $d r$ contains in cycle $t^{\prime}+1$ the page table entry needed for the translation of $v a$. If ptea $\left(h^{t s}, v a\right)[2]=1$, it is in the upper half of the data register. Otherwise, it is in the lower half.

$$
p t e\left(h^{t s}, v a\right)= \begin{cases}d r^{t^{\prime}+1}[63: 32] & \text { if ptea }\left(h^{t s}, v a\right)[2]=1 ; \\ d r^{t^{\prime}+1}[31: 0] & \text { otherwise. }\end{cases}
$$

One cycle later the MMU's address register ar contains the translated address

$$
a r^{t^{\prime}+2}=\operatorname{pma}\left(h^{t s}, v a\right)
$$

Consider the cycle $t^{\prime \prime} \geq t^{\prime}+2$ when control is in state read and the busy signal from the memory interface is zero. Using $G 4$ one argues that the memory output $m$ dout contains in cycle $t e=t^{\prime \prime}+1$ the required result of the translated read access

$$
\left.p . d o u t^{t e}=m . d o u t^{t e}=m\left(h^{t s}\right)_{8}\left(p m a\left(h^{t s}, v a \circ 0^{3}\right)\right)\right)
$$

\section{Guaranteeing the operating conditions}

Stable inputs from the CPU to the MMU's (Condition $G 1$ ) can be guaranteed by using the stabilizer circuits mentioned in Section 4.2 with very modest enhancements. Condition $G 4$ for loads can be guaranteed, if loads and stores are performed in order by the memory unit. Guaranteeing the remaining operating conditions (Conditions $G 2, G 3$, and $G 4$ for fetch) requires a software convention and a hardware construction. 


\subsection{Software Synchronization Convention}

We consider sequential computations of the physical machine $\left(c_{P}^{0}, c_{P}^{1}, \ldots\right)$; formally this means that we have for all steps $i$ :

$$
c_{P}^{i}=\delta_{P}\left(c_{P}^{i-1}, e e v^{i}\right)
$$

Recall that for physical machines the address $i a d d r\left(c_{P}\right)$ from which an instruction is fetched depends on $c_{P}$.mode:

$$
\operatorname{iaddr}\left(c_{P}\right)= \begin{cases}c_{P} \cdot D P C & \text { if } c_{P} . \text { mode }=0 ; \\ p m a\left(c_{P}, c_{P} \cdot D P C\right) & \text { otherwise. }\end{cases}
$$

The instruction $I\left(c_{P}\right)$ executed in configuration $c_{P}$ is then

$$
I\left(c_{P}\right)=c_{P} \cdot p m_{4}\left(i a d d r\left(c_{P}\right)\right)
$$

We define an instruction as synchronizing if it is completed and the pipeline of the processor is drained before the (translation of the) fetch of the sequentially next instruction starts. The VAMP processor has already a synchronizing instruction, namely a movs2i instruction with $I E E E f$ as a source register. ${ }^{4}$ We now also define the $r f e$ instruction to be synchronizing. With the help of function $I\left(c_{P}\right)$ one defines a predicate $\operatorname{syncing}\left(c_{P}\right)$ stating that the instruction executed in configuration $c_{P}$ is synchronizing. The software sync-convention now has two parts:

1. Let $t<t^{\prime}$. Assume $I\left(c_{P}^{t}\right)$ writes to $i a d d r\left(c_{P}^{t^{\prime}}\right)$. Then for some $t^{\prime \prime}$ between $t$ and $t^{\prime}$ instruction $I\left(c_{P}^{t^{\prime \prime}}\right)$ must be synchronizing, i.e. we have syncing $\left(c_{P}^{t^{\prime \prime}}\right)$. The corresponding condition is also needed without address translation in order to prevent the modification of an instruction in pipelined machines after it has been (pre-) fetched $\left[\mathrm{SH} 98, \mathrm{BJK}^{+} 03\right]$.

2. Let $t<t^{\prime}$. Assume instruction $I\left(c_{P}^{t^{\prime}}\right)$ is in user mode $\left(c_{P}^{t^{\prime}} \cdot \operatorname{mode}=1\right)$ and instruction $I\left(c_{P}^{t}\right)$ writes a page table entry read during the fetch of $I\left(c_{P}^{t^{\prime}}\right)$; the address of this entry is ptea $\left(c_{P}^{t} . D P C\right)$ for fetch. Then we also require syncing $\left(c_{P}^{t^{\prime \prime}}\right)$ for an instruction $t^{\prime \prime}$ between $t$ and $t^{\prime}$.

Clearly, the first sync-convention addresses operating conditions $G 4$ in case of a fetch, whereas sync Condition 2 addresses 63 . In the hardware one has to address operating condition $G 2$ and one has to implement the flushing of the processor once a synchronizing instruction is decoded.

\subsection{Hardware mechanisms for synchronization}

The VAMP processor has a two stage pipeline for instruction fetch and instruction decode, followed by a Tomasulo scheduler. For details see $\left[\mathrm{BJK}^{+} 03, \mathrm{Kro01}, \mathrm{Bey04}\right]$. Thus, there are many register stages $S$, e.g. $I F$ for instruction fetch, $P C s$ with $P C$ and $D P C$, ID for instruction decode, $R S(r s)$ for reservation station $r s, R O B(t a g)$ for the content of the reorder buffer with address tag, $R F$ for the register files, etc. In particular the instruction register $I$ belongs to stage $I D$.

The clocking and stalling of individual stages is achieved by a so-called stall engine. For an introduction to stall engines see [MP00]; for better stall engines see [Kro01, Bey04]. We enhance here the stall engine from [Bey04].

\footnotetext{
${ }^{4}$ This instruction reads out the floating point interrupts accumulated so far.
} 
Three crucial data structures resp. signals are associated with each stage $S$ in the stalling engine:

1. The full bit full $_{S}$. It is on, if stage $S$ has meaningful data. Clearing the bit $\mathrm{full}_{S}$ flushes the stage. Here we will only be concerned with bit $f_{u l l}$ ID of the instruction decode stage.

2. The local busy signal busys. If this signal is on in cycle $t$, then the circuits with inputs from register stage $S$ do not produce meaningful data at the end of cycle $t$. Here we will only be concerned with the busy signal busy $y_{I F}$ of the instruction fetch stage.

3. The update enable signals $u e_{S}$. It is like a clock enable signal. If $u e_{S}$ is active in cycle $t$, the stage $S$ has new data in cycle $t+1$. We will use these signals in Section 6.1 for the definition of scheduling functions.

Let busy $y_{I F}^{\prime}$ be the busy signal of the instruction fetch stage of a machine without memory management units. We define a new busy signal by

$$
\operatorname{busy}_{I F}(h)=\operatorname{busy}_{I F}^{\prime}(h) \vee \neg f e t c h(h)
$$

where signal $f e t c h(h)$ is almost the read signal for the instruction MMU of the CPU. ${ }^{5}$

Signal fetch is turned on, if (i) no instruction changing registers pto, $p t l$ and mode is in progress and (ii) no synchronizing instruction is in progress. Instructions in progress can be in the instruction decode stage, i.e. in the instruction register $I R$, or they are issued but not completed, thus they are in the Tomasulo scheduler and its data structures. In a Tomasulo scheduler an instruction in progress which changes a register $r$ from a register file is easily recognized by an inactive valid bit $r . v$. Thus we define

$$
\text { fetch }(h)=\text { h.pto.v } \wedge \text { h.ptl.v } \wedge \text { h.mode.v } \wedge \text { fetch }^{\prime}(h)
$$

where function $\mathrm{fetch}^{\prime}(h)$ has to take care of instructions in the decode stage. Using predicates like $r f e()$ which are already defined for configurations also for the contents of the instruction register, we set

$$
\text { fetch }^{\prime}(h)=\neg\left(h . \text { full }_{I D} \wedge(\operatorname{syncing}(I R) \vee \operatorname{movi} 2 s(I R) \vee r f e(I R))\right) .
$$

In the VAMP processor synchronizing instructions stay in the instruction decode stage until the can immediately proceed to the write-back stage.

\section{Processor correctness}

\subsection{Correctness criteria}

We are using correctness criteria based on scheduling functions from [MP00, Kro01, Bey04, SH98]. Register stages $S$ of the hardware come in three flavours:

- Visible stages (with respect to the physical machine): these stages are (i) $P C s$ with the program counters h.PC, h.DPC, (ii) $R F$ with the register files and h.GPR, h.SPR, and h.FPR (iii) stage $\mathrm{mem}^{\prime}$

\footnotetext{
${ }^{5}$ It is latched for the completion of an interrupted access
} 
with the user visible memory. This latter stage does not exist directly as a hardware component; instead it is simulated by the memory system (main memory and caches) and its state in hardware configuration $h$ is encoded in function $m(h)$ (cf. Section 4.2).

- Invisible stages: the registers of these stages store values of auxiliary functions used in the definition of the sequential semantics of the physical machines. E.g. stage $I D$ with the instruction register stores values $I\left(c_{P}\right)$, stage mem with the input registers to the memory system for load / store operations stores $e a\left(c_{P}\right)$, and so on.

- Stages from the data structures of the Tomasulo scheduler.

We map hardware stages $S$ and hardware cycles $t$ to instruction numbers $i$ by means of scheduling functions: $s I(S, t)=i$. The intention is to relate configurations $h^{t}$ of the hardware with configurations $c_{P}^{i}$ of the specification machines in the following way:

1. For visible registers $R$ from stages $S \neq$ mem' $^{\prime}$ :

$$
h^{t} \cdot R=c_{P}^{s I(t, S)} \cdot R
$$

Thus the specified value of visible hardware register $R$ is the same as the value of $R$ in the specification machine before execution of the $i$ 'th instruction, where $i=s I(S, t)$ is the instruction scheduled in stage $S$ during cycle $t$.

The next two condition differ purely by notation:

2. For stage $\mathrm{mem}^{\prime}$ :

$$
m\left(h^{t}\right)=c_{P}^{s I\left(m e m^{\prime}, t\right)} \cdot p m
$$

3. For invisible registers $R$ in stage $S$ :

$$
h^{t} \cdot R=R\left(c_{P}^{s I(S, T)}\right)
$$

4. Specific correctness criteria are used for the data structures of the Tomasulo scheduler. For details see [Kro01].

The three main definitions for scheduling functions which make this work are the following:

1. In order fetch: The fetch scheduling function is incremented if instruction decode stage receives a new instruction. Recall that $u e_{I D}$ is the update enable function of the instruction register:

$$
s I(\text { fetch }, t+1)= \begin{cases}s I(\text { fetch }, t)+1 & \text { if } u e_{I D}^{t} ; \\ s I(\text { fetch }, t) & \text { otherwise. }\end{cases}
$$

2. The scheduling of a stage $S^{\prime}$ that is not updated does not change:

$$
u e_{S^{\prime}}^{t}=0 \rightarrow s I\left(S^{\prime}, t+1\right)=s I\left(S^{\prime}, t\right)
$$

3. If data are clocked in cycle $t$ from stage $S^{\prime}$ to $S$ (a formal definition depends on update enable signals, mux select signals, or driver enable signals during cycle $t$ ) then

$$
s I\left(S^{\prime}, t+1\right)= \begin{cases}s I(S, t)+1 & \text { if } S^{\prime} \text { invisible; } \\ s I(\text { fetch }, t) & \text { if } S^{\prime} \text { visible. }\end{cases}
$$

Thus intuitively an instruction number $i=s I(S, t)$ moves together with the data through the datapath; upon reaching a register in a visible stage $S^{\prime}$ however, the register receives the value after the $i$-th instruction, i.e. before instruction $(i+1)$-th instruction. 


\subsection{Correctness proof with external interrupt sig- nals}

In general the hardware of a pipelined processor does not complete one instruction per cycle. As there are more cycles $t$ than instructions $i$ there are necessarily more external interrupt events signals eev $v_{h}^{t}$ 'seen' by the hardware than event signals $e e v^{i}$ seen by the sequential specification machine. As the computation of the sequential machine is defined by

$$
c_{P}^{i+1}=\delta_{P}\left(c_{P}^{i}, e e v^{i}\right)
$$

one has to define the interrupt signal $e e v^{i}$ seen by the specification machine from the signals $e e v_{h}^{t}$ seen by the hardware and the processor. This has already been observed in [SH98, MP00].

The VAMP processor samples external interrupt signals only during the write-back stage $W B$ (i.e. it does not sample them all). Every instruction $i$ is only for one cycle $t$ in the write-back stage. Call this cycle $t=W B(i)$. The correctness proof then works with

$$
e e v^{i}=e e v_{h}^{W B(i)}
$$

That no harm is done by the external interrupts signals, which are ignored both by the sampling of the hardware and the sequential programming model, is a problem that has to be solved by the protocol between the processor and the I/O devices.

\subsection{Correctness proof}

We give the new part of the processor correctness proof for a translated instruction fetch without exceptions. The other new cases are handled by similar arguments. Thus consider a translated read access on the instruction port of the $\mathrm{CPU}$ which lasts from cycle $t s$ to cycle te, let $i=s I(f e t c h, t s)$ and let $t \in\{t s, \ldots, t e\}$ be any cycle of the access. From the correctness proof for the processor hardware we conclude, that on the address bus of the instruction MMU PI.addr we have observed during cycle $t$ the correct delayed PC,

$$
P I . a d d r\left(h^{t}\right)=c_{P}^{i} \cdot D P C[31: 2] .
$$

Let $i^{\prime}(t)=s I(R F, t)<i$ be the instruction in the register stage during cycle $t$. Also by processor correctness we have for all registers $R$ of the register files

$$
h^{t} \cdot R=c_{P}^{i^{\prime}(t)} \cdot R
$$

In particular this holds for registers $R \in\{p t o, p t l$, mode $\}$. By the construction of the fetch signal all instructions $x<i$ that update special purpose register $p t o, p t l$, or mode have already left the pipe already at cycle $t s$ (and, because we fetch in order no instructions $x>i$ can enter the pipe while instruction $i$ is in the fetch stage). We conclude for registers $R \in\{p t o, p t l$, mode $\}$ and all cycles $t \in\{t e, \ldots, t s\}$ that

$$
c_{P}^{i} \cdot R=c_{P}^{i^{\prime}(t)} \cdot R=h^{t} \cdot R
$$

Let $i^{\prime \prime}(t)=s I\left(\mathrm{mem}^{\prime}, t\right)$. From processor correctness we get

$$
m\left(h^{t}\right)=c_{P}^{i^{\prime \prime}(t)} \cdot p m
$$


By the second part of the software sync-convention all instructions $x<i$ which write the page table entry address $p t e a\left(c_{P}^{i}, c_{P}^{i} . D P C\right)$ have left the pipe already at cycle $t s$. We conclude

$$
\begin{aligned}
\operatorname{pte}\left(c_{P}^{i}, c_{P}^{i} \cdot D P C\right) & =c_{P}^{i} \cdot p m_{4}\left(p t e a\left(c_{P}^{i}, c_{P}^{i} \cdot D P C\right)\right) \\
& =c_{P}^{i^{\prime \prime}(t)} \cdot p m_{4}\left(p t e a\left(c_{P}^{i}, c_{P}^{i} \cdot D P C\right)\right) \\
& =m\left(h^{t}\right)_{4}\left(p t e a\left(c_{P}^{i}, c_{P}^{i} \cdot D P C\right)\right)
\end{aligned}
$$

By the first part of the software sync-convention all instructions which write the physical memory address $\operatorname{pma}\left(c_{P}^{i}, c_{P}^{i} . D P C\right)$ have also left the pipe already at cycle $t s$. As above we conclude

$$
\begin{aligned}
I\left(c_{P}^{i}\right) & =c_{P}^{i} \cdot p m_{4}\left(p m a\left(c_{P}^{i}, c_{P}^{i} \cdot D P C\right)\right) \\
& =c_{P}^{i^{\prime \prime}(t)} \cdot p m_{4}\left(p m a\left(c_{P}^{i}, c_{P}^{i} \cdot D P C\right)\right) \\
& =m\left(h^{t}\right)_{4}\left(p m a\left(c_{P}^{i}, c_{P}^{i} \cdot D P C\right)\right)
\end{aligned}
$$

Hence the operating conditions for the MMU are fulfilled, and we get from the local correctness lemma:

$$
\text { PI.dout }\left(h^{t e}\right)=m\left(h^{t s}\right)_{8}\left(p m a\left(h^{t s}, P I . a d d r\left(h^{t s}\right) \circ 0^{3}\right)\right)
$$

The rest is lengthy but trivial. Specializing the equations above for $t=t s$ gives

$$
\begin{aligned}
\text { PI.addr }\left(h^{t s}\right) & =c_{P}^{i} \cdot D P C[31: 2] \\
h^{t s} \cdot p t o & =c_{P}^{i} \cdot p t o \\
p t e\left(c_{P}^{i}, c_{P}^{i} \cdot D P C\right) & =m\left(h^{t s}\right)_{4}\left(p t e a\left(c_{P}^{i}, c_{P}^{i} \cdot D P C\right)\right) \\
I\left(c_{P}^{i}\right) & =m\left(h^{t s}\right)_{4}\left(p m a\left(c_{P}^{i}, c_{P}^{i} \cdot D P C\right)\right)
\end{aligned}
$$

Using the definition of functions ptea, pte and pma for hardware machines we conclude successively

$$
\begin{aligned}
& \text { ptea }\left(h^{t s}, c_{P}^{i} \cdot D P C\right)=h^{t s} \cdot p t o+4 \cdot c_{P}^{i} \cdot D P C \cdot p x \\
& =c_{P}^{i} \cdot p t o+4 \cdot c_{P}^{i} \cdot D P C \cdot p x \\
& =\operatorname{ptea}\left(c_{P}^{i}, c_{P}^{i} \cdot D P C\right) \\
& p t e\left(h^{t s}, c_{P}^{i} \cdot D P C\right)=m\left(h^{t s}\right)_{4}\left(p t e a\left(h^{t s}, c_{P}^{i} \cdot D P C\right)\right) \\
& =m\left(h^{t s}\right)_{4}\left(\text { ptea }\left(c_{P}^{i}, c_{P}^{i} \cdot D P C\right)\right) \\
& =p t e\left(c_{P}^{i}, c_{P}^{i} \cdot D P C\right) \\
& p m a\left(h^{t s}, c_{P}^{i} \cdot D P C\right)=p t e\left(h^{t s}, c_{P}^{i} \cdot D P C\right) \cdot p x \circ c_{P}^{i} \cdot D P C \cdot b x \\
& =p t e\left(c_{P}^{i}, c_{P}^{i} \cdot D P C\right) \cdot p x \circ c_{P}^{i} \cdot D P C \cdot b x \\
& =\operatorname{pma}\left(c_{P}^{i}, c_{P}^{i} \cdot D P C\right) \\
& P I . d o u t\left(h^{t e}\right)=m\left(h^{t s}\right)_{8}\left(p m a\left(h^{t s}, P I . a d d r\left(h^{t s}\right) \circ 0^{3}\right)\right) \\
& =m\left(h^{t s}\right)_{8}\left(p m a\left(h^{t s}, c_{P}^{i} \cdot D P C[31: 2] \circ 0^{3}\right)\right) \\
& =m\left(h^{t s}\right)_{8}\left(p m a\left(c_{P}^{i}, c_{P}^{i} \cdot D P C[31: 2] \circ 0^{3}\right)\right) \\
& =c_{P}^{i} \cdot p m_{8}\left(p m a\left(c_{P}^{i}, c_{P}^{i} \cdot D P C[31: 2] \circ 0^{3}\right)\right) \\
& I\left(c_{P}^{i}\right)=c_{P}^{i} \cdot p_{4}\left(p m a\left(c_{P}^{i}, c_{P}^{i} \cdot D P C\right)\right) \\
& = \begin{cases}P I . \operatorname{dout}\left(h^{t s}\right)[63: 32] & \text { if } c_{P}^{i} . D P C[2] ; \\
P I . \operatorname{dout}\left(h^{t s}\right)[31: 0] & \text { otherwise. }\end{cases}
\end{aligned}
$$

Thus, by selecting the right half in the double word PI.dout $\left(h^{\text {te }}\right)$ using bit 2 of the delayed program counter, in cycle $t e+1$ we clock $I\left(c_{P}^{i}\right)$ in the 


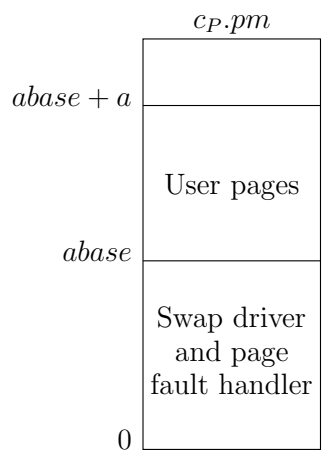

Figure 7: Memory Map with addresses given as page indices.

instruction register $I$. We have $s I(I D, t e+1)=i$. Thus for a translated fetch without exceptions lasting from cycle ts to cycle te we have shown the required hardware correctness statement

Lemma $3 h^{t e+1} \cdot I=I\left(c_{P}^{i}\right)=I\left(c_{P}^{s I(I D, t e+1)}\right)$

\section{$7 \quad$ Virtual machine simulation}

In this section we outline an informal proof that a physical machine with an appropriate page fault handler can simulate a virtual machine. We will use pseudo code as well as C like data structures in order to describe the handler and we will argue in the style of an efficient algorithms paper. Making these arguments precise is not trivial; we give some details in the section on further work.

For page indices $p x$ we define the physical page $p x$, the swap page $p x$, and the virtual page $p x$ respectively as

$$
\begin{aligned}
& \operatorname{ppage}\left(c_{P}, p x\right)=c_{P} \cdot p m_{4 K}\left(p x \circ 0^{12}\right), \\
& \operatorname{spage}\left(c_{P}, p x\right)=c_{P} \cdot s m_{4 K}\left(p x \circ 0^{12}\right), \text { and } \\
& \operatorname{vpage}\left(c_{V}, p x\right)=c_{V} \cdot v m_{4 K}\left(p x \circ 0^{12}\right) .
\end{aligned}
$$

We extend the definition of physical page indices $p p x\left(c_{P}, v a\right)$ and valid bits $v\left(c_{P}, v a\right)$ from addresses $v a$ to page indices $p x$ by

$$
\begin{aligned}
p p x\left(c_{P}, p x\right) & =p p x\left(c_{P}, p x \circ 0^{12}\right) \text { and } \\
v\left(c_{P}, p x\right) & =v\left(c_{P}, p x \circ 0^{12}\right) .
\end{aligned}
$$

\subsection{Memory map of the physical machine}

We partition the physical memory $c_{P} . p m$ into user memory and system memory according to Figure 7 . Starting at the physical address with page index abase we allocate $a$ pages of user memory. This defines the set of user page indices

$$
U P=\{u \mid \text { abase } \leq\langle u\rangle<\text { abase }+a-1\} .
$$

Physical addresses with page indices smaller than abase are used by the page fault handler and the swap memory driver.

We list below the data structures used by the handler and some invariants for them. 
- A process control block $P C B$ to save the processor registers of the virtual processor when the processors runs in system mode.

- The page table

$$
P T\left(c_{P}\right)=c_{P} \cdot p m_{4 \cdot V}\left(c_{P} \cdot p t o \cdot 4 K\right)
$$

where $V=\left\langle c_{P} . p t l\right\rangle+1$ is the number of accessible virtual pages. We require for all virtual page indices $0 \leq p x<V$ that the corresponding physical page index belongs to a user page if it is valid,

$$
v\left(c_{P}, p x\right) \Longrightarrow p p x\left(c_{P}, p x\right) \in U P \text {. }
$$

- the physical page index $M R L$ of the most recently loaded page.

- A swap page index sbase in swap memory. For virtual addresses va we will use the swap memory address

$$
\operatorname{sma}\left(c_{P}, v a\right)=\text { sbase } \cdot 4 K+v a
$$

- A user page index $b \in\{0, \ldots, a-1\}$. We call a user page $u \in$ $\{0, \ldots, a-1\}$ full if it stores a valid virtual page, i.e. if there is a virtual page index $0 \leq p x<V$ such that

$$
v\left(c_{P}, p x\right) \wedge p p x\left(c_{P}, p x\right)=a b a s e+u
$$

A user page which is not full is called free. We maintain as an invariant that user page $u$ is free iff $u>b$.

- an array $B$ of size $a$ holding virtual page indices. If $u \leq b$, i.e. if user page $u$ is full, then

$$
\text { abase }+u=p p x\left(c_{P}, B[u]\right)
$$

This data structure is used for victim selection, if a page has to be evicted.

- Parameters $p p x p, s p x p$, and $p 2 s$ of the driver for the swap memory.

\subsection{Simulation relation}

For virtual machine configurations $c_{V}$ and physical machine configurations $c_{P}$ we define a simulation relation $B\left(c_{P}, c_{V}\right)$ stating that configuration $c_{P}$ is an encoding for configuration $c_{V}$. We require that the invariants of the previous subsections hold for the physical machine and that the physical machine is in user mode $\left(c_{V} \cdot\right.$ mode $\left.=1\right)$. The simulation relation is composed of two parts:

1. For all virtual addresses $v a$

$$
c_{V} \cdot p(v a)=p\left(c_{P}, v a\right) .
$$

i.e. the write protection function is encoded in the protection bits of the page tables.

2. For all virtual addresses $v a$

$$
c_{V} \cdot v m(v a)= \begin{cases}c_{P} \cdot p m\left(p m a\left(c_{P}, v a\right)\right) & \text { if } v\left(c_{P}, v a\right) ; \\ c_{P} \cdot s m\left(\operatorname{sma}\left(c_{P}, v a\right)\right) & \text { otherwise. }\end{cases}
$$

So, the user pages of physical memory act as a (write-back) cache for the swap memory. This condition may be equivalent formulated for all virtual page indices $p x$ as

$$
\operatorname{vpage}\left(c_{V}, p x\right)= \begin{cases}\operatorname{ppage}\left(c_{P}, p p x\left(c_{P}, p x\right)\right) & \text { if } v\left(c_{P}, p x\right) \\ \operatorname{spage}\left(c_{P}, \operatorname{sbase}+p x\right) & \text { otherwise }\end{cases}
$$




\subsection{Page fault handler and software conditions}

Assume the interrupt occurred during physical machine configuration $c_{P}$ encoding virtual machine configuration $c_{V}$, i.e. we have $B\left(c_{P}, c_{V}\right)$.

We describe a very simple handler; the handler itself is never interrupted. Thus it suffices that the handler saves only the general purpose registers of the physical processor into the process control block. Testing the exception cause register ECA it is easy to determine, whether a page fault on fetch or a page fault on load store has occurred. For the former we have $E C A[3]=1$, for the latter we have $E C A[4]=1$.

It is easy to specify and implement the handler in case of an exception due to page table length exception or rights violations: the exception is recognized and the simulation is stopped. Thus assume a page fault occurs, because the required virtual page is not in physical memory. The virtual address $x v a$ causing the exception is defined as

$$
x v a= \begin{cases}E D P C & \text { if } p f f ; \\ E D A T A & \text { if } p f l s .\end{cases}
$$

We call the page index of the exception virtual address the exception virtual page $x v=x v a . p x$. Because this page is not in memory and $B\left(c_{P}, c_{V}\right)$ holds, we know that the correct virtual page is stored in swap memory

$$
\operatorname{spage}\left(c_{P}, \text { sbase }+x v\right)=\operatorname{vpage}\left(c_{V}, x v\right)
$$

If $b=a-1$ there are no free user pages and a victim physical page index $v p$ is selected from the user pages. However, the most recently loaded page is never chosen as victim to avoid deadlock, so $v p \in U P \backslash\{M R L\}$.

Let $v p=a b a s e+u$. By looking up table entry $B[u]$ we determine the corresponding victim virtual page index $v v=B[u]$ of the virtual page stored at physical page $v p$.

Because $B\left(c_{P}, c_{V}\right)$ holds and $p p x\left(c_{P}, v v\right)=a b a s e+u$ we have

$$
\begin{aligned}
\operatorname{vpage}\left(c_{V}, v v\right) & =\operatorname{ppage}\left(c_{P}, \operatorname{ppx}\left(c_{P}, v v\right)\right) \\
& =\operatorname{ppage}\left(c_{P}, \operatorname{ppx}\left(c_{P}, B[u]\right)\right) \\
& =\operatorname{ppage}\left(c_{P}, \text { abase }+u\right) \\
& =\operatorname{ppage}\left(c_{P}, v p\right)
\end{aligned}
$$

Running the driver with parameters

$$
(\text { ppxp, spxp, } p 2 s)=(v p, s b a s e+v v, 1)
$$

will save the victim page to swap memory, i.e. we end up in a physical machine configuration $c_{P}^{(1)}$ with

$$
\operatorname{spage}\left(c_{P}^{(1)}, \text { sbase }+v v\right)=\operatorname{ppage}\left(c_{P}, v p\right) .
$$

We conclude

$$
\operatorname{spage}\left(c_{P}^{(1)}, \operatorname{sbase}+v v\right)=\operatorname{ppage}\left(c_{P}, v p\right)=\operatorname{vpage}\left(c_{V}, v v\right) .
$$

Hence, if we clear the valid bit of page $v v$ and enter a configuration $c_{P}^{(2)}$ with $v\left(c_{P}^{(2)}, v v\right)=0$, the simulation relation $B\left(c_{P}^{(2)}, c_{v}\right)$ still holds.

We now have a user page index $e$ where we can swap in the exception virtual page, namely

$$
e= \begin{cases}\text { abase }+b+1 & \text { if } b<a-1 \\ v p & \text { otherwise }\end{cases}
$$


In the first case we increment $b$. Running the driver with parameters

$$
(\text { ppxp, spxp, } 2 s)=(e, s b a s e+x v, 0)
$$

will swap the exception virtual page into physical memory, i.e. we end up in a configuration $c_{P}^{(3)}$ where

$$
\begin{aligned}
\operatorname{ppage}\left(c_{P}^{(3)}, e\right) & =\operatorname{spage}\left(c_{P}, \operatorname{sbase}+x v\right) \\
& =\operatorname{vpage}\left(c_{V}, x v\right) .
\end{aligned}
$$

Thus, $B\left(c_{P}^{(4)}, c_{V}\right)$ and the invariants will hold if we set

$$
\begin{aligned}
v\left(c_{P}^{(4)}, v x\right) & =1 \\
p p x\left(c_{P}^{(4)}, v x\right) & =e \\
B[e-a b a s e] & =v x \\
M R L & =e
\end{aligned}
$$

in a later configuration $c_{P}^{(4)}$. The handlers completes its work by restoring the general purpose registers from the process control block and executing an $r f e$ instruction.

By inspection of the handler we see that the software synchronization conditions are fulfilled and thus we can conclude that with this handler the hardware specified above will work correctly.

\subsection{Simulation theorem}

Theorem 1 For every computation $\left(c_{V}^{0}, c_{V}^{1}, \ldots\right)$ of the virtual machine there is a computation $\left(c_{P}^{0}, c_{P}^{1}, \ldots\right)$ of the physical machine and there are step numbers $(s(0), s(1), \ldots)$ such that we have for all $t$ :

$$
B\left(c_{V}^{t}, c_{P}^{s(t)}\right)
$$

This means that $t$ steps of the virtual machine are simulated after $s(t)$ steps by the physical machine. This is obviously shown by induction on $t$. For the initialization we set $b=-1$, i.e. all physical page are invalid, and the entire virtual memory is stored in swap memory. Concluding from $t$ to $t+1$ there are several cases. If there is no exception, then one step of the virtual machine is simulated by one step of the physical machine running in user mode ${ }^{6}$ and we get

$$
s(t+1)=s(t)+1
$$

Because in a single instruction we can have up to two page faults, there remain four cases:

1. A single page fault on fetch.

2. A single page fault on load / store.

3. A page fault on fetch followed by a page fault on load / store.

4. A page fault on load / store followed by a page fault on fetch. This case occurs if the page with the current instruction is the victim page of the first page fault.

${ }^{6}$ Not in one cycle of the hardware! 
For physical machine steps $s$ let $\tau(s)$ be the first step $s^{\prime}$ after $s$ such that the machine is in user mode:

$$
\tau(s)=\min \left\{s^{\prime} \mid s^{\prime}>s \wedge c_{P}^{s^{\prime}} . \operatorname{mode}\right\}
$$

In all four cases we have a page fault in step $s(t)+1$. In the first two cases the simulation of step $t+1$ succeeds without page fault in the first step in user mode after step $s(t)+1$ thus

$$
s(t+1)=\tau(s(t)+1)+1 .
$$

In the other two cases the second page fault occurs in step

$$
s^{\prime}=\tau(s(t)+1)+1
$$

Because the victim page of the second page fault is not the page swapped in during the handling of the first page fault the simulation will succeed in step

$$
s(t+1)=\tau\left(s^{\prime}\right)+1
$$

\section{Summary and further work}

We have presented two main results. (i) We have reported about the formal verification of a processor with (simple) MMUs. The local correctness proof for an MMU alone (Section 4.4) is straight forward, but it hinges on nontrivial operating conditions. Guaranteeing the operating conditions requires a variety of arguments, from very detailed arguments about the hardware (e.g. Section 5.2) to the format of page fault handlers (Section 7.3). (ii) Arguing about low level system software we have given a paper and pencil proof for a simulation theorem stating that virtual machines are simulated by physical machines with appropriate page fault handlers. Because modern operating systems support multitasking and virtual memory, the results of this paper are crucial steps towards the verification of entire computers systems.

Presently we see three main directions for further work. (i) On the hardware side one wants to verify processors with pipelined MMUs, multi level page tables and table look aside buffers. (ii) On the low level software side one wants to formally prove the simulation theorem of this paper. This is part of an ongoing effort to formally verify an entire operating system kernel as part of the Verisoft project. Paper and pencil proofs can be found in [SysArch04] (iii) one wants to establish correctness theorems for the memory management mechanisms of shared memory multiprocessors. The thesis [Hil05] contains results of this nature.

\section{References}

[ACHK04] Mark Aagaard, Vlad Ciubotariu, Jason Higgins, and Farzad Khalvati. Combining equivalence verification and completion functions. In Alan $\mathrm{Hu}$ and Andrew Martin, editors, FMCAD, volume 3312 of Lecture Notes in Computer Science, pages 98112. Springer, 2004.

[Bev89] William R. Bevier. Kit and the short stack. Journal of Automated Reasoning, 5(4):519-530, 1989. In [Boy89]. 
[Bey04] Sven Beyer. Putting It All Together: Formal Verification of the $V A M P$. PhD thesis, Saarland University, Computer Science Department, 2004.

$\left[\mathrm{BJK}^{+} 03\right]$ Sven Beyer, Christian Jacobi, Daniel Kroening, Dirk Leinenbach, and Wolfgang Paul. Instantiating uninterpreted functional units and memory system: functional verification of the VAMP processor. In Daniel Geist and Enrico Tronci, editors, CHARME, volume 2860 of Lecture Notes in Computer Science, pages 51-65. Springer, 2003.

[BM88] Robert S. Boyer and J S. Moore. A Computational Logic Handbook. Academic Press, 1988.

[Boy89] Robert S. Boyer, editor. Special Issue on System Verification, volume 5 of Journal of Automated Reasoning. Kluwer Academic Publishers, 1989.

[Hil05] Mark Hillebrand. Address Spaces and Virtual Memory: Specification, Implementation, and Correctnesss (Draft). PhD thesis, Saarland University, Saarbrücken, Germany, 2005.

[Hun89] Warren A. Hunt. Microprocessor design verification. Journal of Automated Reasoning, 5(4):429-460, December 1989. In [Boy89].

[Kro01] Daniel Kroening. Formal Verification of Pipelined Microprocessors. PhD thesis, Saarland University, Computer Science Department, 2001.

[Moo89] J Strother Moore. A mechanically verified language implementation. Journal of Automated Reasoning, 5(4):461-492, 1989. In [Boy89].

[Moo03] J Strother Moore. A grand challenge proposal for formal methods: A verified stack. In Bernhard K. Aichernig and T. S. E. Maibaum, editors, 10th Anniversary Colloquium of UNU/IIST, volume 2757 of Lecture Notes in Computer Science, pages 161-172. Springer, 2003.

[MP00] Silvia M. Mueller and Wolfgang J. Paul. Computer Architecture: Complexity and Correctness. Springer, 2000.

[OSR92] S. Owre, N. Shankar, and J. M. Rushby. PVS: A prototype verification system. In 11th International Conference on Automated Deduction (CADE), volume 607 of Lecture Notes in Artificial Intelligence, pages 748-752. Springer, 1992.

[SH98] Jun Sawada and Warren A. Hunt, Jr. Processor verification with precise exeptions and speculative execution. In $C A V$ '98: Proceedings of the 10th International Conference on Computer Aided Verification, pages 135-146. Springer-Verlag, 1998.

[Ver03] The Verisoft Project. http://www.verisoft.de/, 2003.

[You89] William D. Young. A mechanically verified code generator. Journal of Automated Reasoning, 5(4):493-518, 1989. In [Boy89]. 